COVER SHEET

To reference this article, please use the following citation:

Jabbal, M. and Zhong, S. (2008). The near wall effect of synthetic jets in a boundary layer. International Journal of Heat and Fluid Flow. Vol. 29, No. 1, pp. 119-130. 


\title{
THE NEAR WALL EFFECT OF SYNTHETIC JETS IN A BOUNDARY LAYER
}

\author{
M. Jabbal ${ }^{*}$ and S. Zhong \\ University of Manchester, School of Mechanical, Aerospace and Civil Engineering, \\ Goldstein Laboratory, Barton Airport, Manchester M30 7RU, UK
}

\section{ABSTRACT}

An experimental investigation to analyse the qualitative near wall effect of synthetic jets in a laminar boundary layer has been undertaken for the purpose of identifying the types of vortical structures likely to have delayed separation on a 2D circular cylinder model described in this paper. In the first instance, dye visualisation of the synthetic jet was facilitated in conjunction with a stereoscopic imaging system to provide a unique quasi three-dimensional identification of the vortical structures. Secondly, the impact of synthetic jet structures along the wall was analysed using a thermochromic liquid crystal-based convective heat transfer sensing system in which, liquid crystals change colour in response to the thermal footprints of a passing flow structure. Of the different vortical structures produced as a result of varying actuator operating and freestream conditions, the footprints of hairpin vortices and stretched vortex rings revealed a marked similarity with the oil flow pattern of a vortex pair interacting with the separation line on the cylinder hence suggesting that either of these structures was responsible in delaying separation. Conditions were established for the formation of the different synthetic jet structures in non-dimensional parameter space.

Keywords: Synthetic jet in boundary layer; Flow control; Vortex structures; Near wall

\footnotetext{
* Corresponding author: Email address: mark_jabbal@hotmail.com
} 


\section{NOMENCLATURE}

$c_{f} \quad$ skin friction coefficient

d boundary layer thickness to orifice diameter ratio

$D$ diameter of cavity or orifice, $\mathrm{mm}$

$f \quad$ diaphragm oscillation frequency, $\mathrm{Hz}$

$h \quad$ orifice depth, $\mathrm{mm}$

$H \quad$ cavity height, $\mathrm{mm}$

$L \quad$ dimensionless stroke length

Re Reynolds number

$S \quad$ Stokes number

St Strouhal number

$T$ time period of a diaphragm oscillation cycle, $\mathrm{s}$

$u$ instantaneous velocity, $\mathrm{m} / \mathrm{s}$

$U$ characteristic velocity, $\mathrm{m} / \mathrm{s}$

$V R \quad$ jet-to-freestream velocity ratio

$x \quad$ streamwise distance from orifice exit, $\mathrm{mm}$

$y \quad$ normal distance from orifice exit, $\mathrm{mm}$

\section{Greeks}

$\Gamma \quad$ vortex circulation, $\mathrm{m}^{2} / \mathrm{s}$

$\delta \quad$ boundary layer thickness, $\mathrm{mm}$

$\Delta \quad$ peak-to-peak displacement at the diaphragm centre, $\mathrm{mm}$

$\rho \quad$ fluid density, $\mathrm{kg} / \mathrm{m}^{3}$

$\tau \quad$ surface shear stress, $\mathrm{N} / \mathrm{m}^{2}$

$v \quad$ molecular kinematic viscosity, $\mathrm{m}^{2} / \mathrm{s}$

\section{Superscripts}

- $\quad$ time average

$\sim \quad$ space average

\section{Subscripts}

c cavity value

$j \quad$ jet value

$o \quad$ orifice value

$w \quad$ wall value

$\infty \quad$ freestream value

\section{INTRODUCTION}

Synthetic jet actuators (SJA) provide a novel means of applying flow control and their

potential application for the effective delay of boundary layer separation on aircraft has

been the focus of intense research in recent years (Smith and Glezer, 1998; Crook and

Wood, 2001; Gilarranz and Rediniotis, 2001; Glezer and Amitay, 2002). One of the

reasons for this is due to their unique ability to impart additional momentum on a fluid

region from which it was originally synthesised without a net mass addition, therefore 
requiring no bleed air supply and complex piping. In addition, the oscillatory nature of synthetic jets has been observed to offer greater entrainment of fluid in the near field compared to their continuous counterparts (James et al., 1998; Cater and Soria, 2002).

It is believed that the interaction of the discrete trains of vortices formed out of a SJA (Fig. 1) with a local boundary layer produces streamwise vortical structures, which are capable of delaying flow separation by entraining faster moving fluid from the freestream to the near wall region. Crook and Wood (2001) investigated the flow control effectiveness of an array of SJAs implemented flush to the surface of a circular cylinder upstream of its separation line in a turbulent boundary layer. From Fig. 2, surface oil patterns showed that with the actuators active, the separation line was pushed downstream noticeably. In particular the oil flow revealed the footprints of a welldefined streamwise vortex pair immediately downstream of each synthetic jet that appeared to persist for a long distance downstream and interact with the separated flow. Subsequent dye visualisation by Zhong et al. (2005) has shown that the interaction between a synthetic jet and a boundary layer is complex, varying from hairpin vortices that remain near to the wall to tilted vortex rings that penetrate the boundary layer as the jet-to-freestream velocity ratio increases. It has been hypothesised (Zhong et al., 2005) that the hairpin vortices may have been responsible for the formation of the streamwise vortex pair that interacted with the separation line on the circular cylinder, however further evidence is still to be sought.

In this paper, the results of a qualitative study of the interaction of a synthetic jet with the near-wall region of a laminar boundary layer are presented. As a continuation of the aforementioned investigation, the purpose of the work described herein is to identify the likely type of vortical structures responsible for the delayed separation on the circular cylinder and to establish the conditions that ensure the formation of such 
structures in non-dimensional parameter space. To this end, the nature of vortical structures produced under a range of actuator and freestream conditions are to be investigated with a view to analysing their respective near wall effects where flow control is ultimately desired. The use of a laminar flow provides a logical first step into visualising and understanding the basic mechanism of synthetic jet boundary layer interaction, which would otherwise be difficult to achieve for a turbulent boundary layer. Furthermore, it is believed by the authors that the type of vortical structures produced should essentially be the same as that for a synthetic jet issuing into a turbulent flow with the main difference being in the dissipation rate of the ejected structures near the wall. This study is therefore applicable to turbulent flows, as in the case of the cylinder model described herein.

Realisation of the aims of this study is to be made primarliy through the application of two qualitative visualisation techniques, which are novel to this area of research. In the first instance, dye visualisation of the synthetic jet structures was facilitated in conjunction with a stereoscopic imaging system to allow simultanteous side and surface views. The technique provides a unique quasi three-dimensional identification of the vortical structures produced by the synthetic jet. Secondly, the impact of synthetic jet structures along the wall was analysed using a thermochromic liquid crystal-based convective heat transfer sensing system. Essentially, the liquid crystals change colour in response to the thermal footprints of a flow structure which causes a localised variation of convective heat transfer. The system, similar to that described by Zhong et al. (2000) therefore allows the resident effect of synthetic jets at the wall to be examined where effective flow control is ultimately desired. Collectively, the techniques will provide invaluable insights into both surface and off-surface behaviour related to synthetic jet boundary layer interaction. 


\section{THE EFFECT OF DIMENSIONLESS PARAMETERS ON THE CHARACTERISTICS OF A ROUND SYNTHETIC JET}

\subsection{SJA in Quiescent Conditions}

Smith and Glezer (1998) have shown that for a SJA of a given geometry in quiescent conditions, two independent dimensionless parameters defined using the jet velocity, namely the dimensionless stroke length $L$ and the jet Reynolds number $\operatorname{Re}_{j}$ characterise the synthetic jet flow. According to the slug model (Glezer, 1988) the stroke length $L_{o}$ represents the length of the fluid column expelled during the blowing stroke, i.e.

$$
L=\frac{L_{o}}{D_{o}}=\frac{\bar{U}_{o}}{f D_{o}}
$$

where $\bar{U}_{o}$ is the time-averaged blowing velocity over the entire cycle, which was originally proposed by Smith and Glezer (1998) to facilitate a comparison between synthetic jets and steady jets. For the present study

$$
\bar{U}_{o}=\frac{1}{T} \int_{0}^{T / 2} \tilde{u}_{o}(t) d t=\frac{1}{3} f \Delta\left(\frac{D_{c}}{D_{o}}\right)^{2}
$$

where $\tilde{u}_{o}(t)$ is the instantaneous space-averaged velocity at the orifice exit. Note that from the defintion proposed by Smith and Glezer (1998), $\bar{U}_{o}$ is neither the time average of $\tilde{u}_{o}(t)$ over a whole cycle $T$, which should be zero, nor its time average over half a cycle $T / 2$ (i.e. the blowing half of a cycle), which is $2 \bar{U}_{o}$. Assuming an incompressible flow, Eq. (2) derived by Tang and Zhong (2006) equates the instantaneous volume flow rate displaced by the diaphragm with that expelled through the orifice (where the coefficient has been modified to $1 / 3$ to account for the use of a rubber diaphragm in the present investigation). The jet Reynolds number is defined as

$$
\operatorname{Re}_{j}=\frac{\bar{U}_{o} D_{o}}{v}
$$


A previous study of synthetic jets in quiescent conditions undertaken by the authors (Jabbal et al., 2006) has shown that the Reynolds number based on the stroke length, $\operatorname{Re}_{L}$, (where $\operatorname{Re}_{L}=\operatorname{Re}_{j} L$ ) is directly related to the synthetic jet vortex circulation, $\Gamma / v$ and as such, is a more useful indicator of the vortex strength.

From these two key parameters a third dependent parameter, the Stokes number, $S$, which is also important can be defined accordingly

$$
S=\left(\frac{2 \pi \mathrm{Re}_{L}}{L^{2}}\right)^{1 / 2}
$$

\subsection{SJA in a Cross Flow Boundary Layer}

If the SJA is embedded in a crossflow boundary layer, the list of physical parameters on which the nature of the synthetic jet will depend should be extended to include the local boundary layer thickness $\delta$, the freestream velocity $U_{\infty}$ and the local wall shear stress $\tau_{\mathrm{w}}$. Dimensional analysis gives the following

$$
\pi_{1}=\frac{f D_{o}}{U_{\infty}}, \pi_{2}=\frac{U_{\infty} \delta}{v}, \pi_{3}=\frac{\delta}{D_{o}}, \pi_{4}=\frac{\tau_{w}}{\rho U_{\infty}^{2}}, \pi_{5}=\frac{\bar{U}_{o}}{U_{\infty}}
$$

where $\pi_{1}$ represents the Strouhal number, $S t, \pi_{2}$ the freestream Reynolds number, $\operatorname{Re}_{\delta}, \pi_{3}$ the ratio of boundary layer thickness to orifice diameter, $d, \pi_{4}$ the non-dimensional shear stress (which is equivalent to the skin friction coefficient, $c_{f}=\tau_{w} / 0.5 \rho U_{\infty}{ }^{2}$ ) and $\pi_{5}$ the jet-to-freestream velocity ratio, $V R$.

Combined with the key dimensionless parameters for a SJA in quiescent conditions, that is $L$ and $\mathrm{Re}_{L}$, there is a total of 7 dimensionless parameters. Taking the inter-dependencies of Eq. (6(a)) and (6(b)) into account effectively reduces the number of parameters pertaining to a synthetic jet in a boundary layer to 5 , namely $L, \operatorname{Re}_{L}, V R, d$ and $c_{f}$ 


$$
\begin{gathered}
S t=\frac{V R}{L} \\
\operatorname{Re}_{\delta}=\frac{\operatorname{Re}_{L} \cdot d}{L \cdot V R}
\end{gathered}
$$

\subsection{Discussion on the Effect of the Dimensionless Parameters}

Holman et al. (2005) and Milanovic and Zaman (2005) have shown that there is a threshold of the stroke length for synthetic jet formation, for which $L$ is approximately 0.5 for round synthetic jets in the absence of a crossflow. As such, jet formation and the ensuing injection of vorticity within the boundary layer to promote fluid mixing requires that the stroke length is larger than a critical value. The magnitude of the stroke length also determines the impact from the suction stroke, with a greater influence of suction being asserted on synthetic jets that are formed at low values of $L$ (i.e. those in which the vortical structures have not moved sufficiently far away from the orifice during the blowing stroke). For a synthetic jet issuing into a boundary layer, it is expected that the effect of suction would be confined to the upstream branch of the vortex hence resulting in an asymmetric structure. Furthermore, extensive investigations by Gharib et al. (1998) of the formation of vortex rings generated through piston-driven jets has shown that the flow field generated by large stroke lengths consist of a leading vortex ring followed by a trailing jet. Alternatively, flow fields generated by small stroke lengths show only a single vortex ring. The transition between these two states is observed to occur at a stroke length of approximately 4, thus representing the threshold of the stroke length above which, shedding of secondary vortices occurs due to saturation of vortex circulation within the primary structure. This threshold for round synthetic jets formed in quiescent conditions has also been found to occur at $L=4$ (Jabbal et al., 2006). 
It can be expected that the jet Reynolds number will have an influence on the strength of the vortical structures formed out of the orifice. A previous study by Zhong et al. (2005) of the behaviour of synthetic jets in a laminar boundary layer has characterised the Reynolds number according to the stroke length, $\operatorname{Re}_{L}$, as defined previously. It is believed that the use of $\operatorname{Re}_{L}$ is suitable in quantifying the strength of a vortex ring and is therefore an important quantity where fluid entrainment and mixing in the near wall region via the generation of coherent vorticity is ultimately required. Nevertheless, a prerequisite to vortex strength is that of vortex roll up, which is determined by the Stokes number. It has been shown by Guo and Zhong (2005) that the Stokes number (Eq. (4)) should be greater than 10 to ensure roll up for $L$ less than 4 .

The velocity ratio represents the relative strength between the jet and freestream velocity and characterises the trajectory of the jet through the boundary layer. Milanovic and Zaman (2005) have previously shown that at a given streamwise location, the jet penetration height is solely a function of the momentum flux ratio, which is equivalent to the velocity ratio $V R$, defined in the present study. Thus at low values of $V R$, the synthetic jet will reside within the boundary layer for a longer period of time, thereby increasing its potential effectiveness where flow control is ultimately desired. Zhong et al. (2005) has shown that shear in the boundary layer will have a greater influence on those structures formed at low $V R$ causing vortex stretching, as opposed to those formed at high $V R$. It is worth noting that the level of shear is directly related to the relative size of the boundary layer thickness to the orifice diameter, $d$ and therefore for different $d$, the magnitude of $V R$ should be set accordingly to ensure the trajectory of the synthetic jet remains in the near wall region.

Finally, the skin friction coefficient along the wall, $c_{f}$, is expected to affect the streamwise decay of the vortical structures downstream of the orifice, with the rate of 
vortex dissipation being greater for a higher skin friction (and therefore a higher wall friction velocity). This has particular implications for a synthetic jet embedded in a turbulent flow and will accordingly require augmentation of $L, \operatorname{Re}_{L}$ and $V R$ to ensure similar levels of effectiveness as a synthetic jet in a laminar flow.

Based on the aforementioned discussion, for a SJA of a given geometry embedded in a boundary layer

$$
\text { SJA effectiveness }=f n\left(L, V R, d, \operatorname{Re}_{L}, c_{f}\right)
$$

Where effectiveness is defined as the strength and persistence of fully formed vortical structures in the near wall region of a boundary layer. The functional dependence of the effectiveness of a synthetic jet on the dimensionless parameters given in Eq. (7) also correlates with a scaling law derived by Raju et al. (2005). Using a non-linear regression analysis on data obtained by numerical simulation, a scaling law for the vorticity flux of a synthetic jet was extracted for the case of a zero-pressure gradient laminar boundary layer, in which the vorticity is influenced by a Strouhal number based on the jet velocity (equivalent to $L$ in the present study), a jet to freestream velocity ratio, a boundary layer thickness to slot width ratio and a jet Reynolds number. The scaling law shows that for a constant boundary layer thickness, the vorticity flux of a synthetic jet embedded in a boundary layer varies with the natural logarithm of $\operatorname{Re}_{j}$ and as a linear function of $L$ and $V R^{-1}$

\section{EXPERIMENTAL APPROACH}

Investigation of the interaction of a synthetic jet with a laminar boundary layer was conducted in a tilting water flume at the University of Manchester Goldstein Research Laboratory. The test section is $4 \mathrm{~m}$ in length and has a cross-sectional area of 
$0.3 \mathrm{~m} \times 0.3 \mathrm{~m}$. The maximum freestream velocity in the test section is approximately $0.4 \mathrm{~m} / \mathrm{s}$. The SJA used in this investigation consisted of a cylindrical cavity bounded by rigid sidewalls with an orifice plate at one end and a diaphragm clamped to the other (Fig.1). The cavity has a diameter, $D_{c}=45 \mathrm{~mm}$ and height, $H=25 \mathrm{~mm}$. The orifice has a diameter, $D_{o}=5 \mathrm{~mm}$ and a thickness equivalent to the orifice depth, $h=5 \mathrm{~mm}$.

\subsection{Stereoscopic Dye Visualisation}

For dye flow visualisation, the synthetic jet actuator was mounted on the back of a flat plate that formed the ceiling of the test section, as shown in Fig. 3. The plate constructed from a $5 \mathrm{~mm}$ thick sheet of aluminium, $1.3 \mathrm{~m}$ in length, was mounted horizontally and spans across the width of the test section. The plate has a 1:5 superelliptical leading edge, which according to Narasimha and Prasad (1994), helps to minimise flow separation and premature transition at the leading edge of the plate. The plate was inclined at a small negative incidence (less than $1^{\circ}$ ) to the oncoming flow to ensure that a fully attached laminar boundary layer forms right at the leading edge.

The SJA orifice opening was flush to the lower test surface of the plate and was located approximately $0.7 \mathrm{~m}$ (140 orifice diameters) aft of the leading edge. During testing, the plate and SJA cavity were both fully submerged in water. A diaphragm made from rubber was clamped to the top of the cavity and was attached at its centre via a steel rod to a permanent magnetic shaker housed above water level, which oscillates the diaphragm in a sinusoidal manner. A rubber diaphragm was used to ensure an accurate sine wave deformation, which would otherwise be difficult to achieve with a metallic one for the very low actuation frequencies and amplitudes used in the present investigation. A dye, comprising of a mixture of food colouring and methanol was introduced into the cavity via gravity. 
In the experiment, a Hitachi KP-F120 partial scan CCD camera was used to capture the dye pattern produced by synthetic jets from two orthogonal views simultaneously (from the side and plane of the wall) at a frame rate of $30 \mathrm{fps}$, providing a quasi three-dimensional visualisation of the jet. To facilitate the capture of both views simultaneously onto each image frame a stereoscopic imaging system, as shown in Fig. 4, was set up using a combination of two plane mirrors and an orthogonal prism in accordance with the method described by Zhang et al. (2003).

\subsection{Liquid Crystal Visualisation}

For liquid crystal visualisation, the aluminium plate was replaced with a $10 \mathrm{~mm}$ thick Perspex plate of the same length and width. The plate leading edge profile, incidence of the plate to the oncoming flow and SJA orifice location aft of the leading edge were consistent with those for the dye flow tests. A $500 \mathrm{~mm} \times 250 \mathrm{~mm}$ cavity was cut out of the plate mid-section to accommodate a heater composite insert.

The composite is essentially a flat plate composed of several layers, as shown in Fig. 5(a). The heater is a resistive wire-wound element encased in silicone rubber and is designed to give a uniform heat capacity to a maximum of $1.7 \mathrm{~kW}$. A $3 \mathrm{~mm}$ thick aluminium sheet is attached to the heater to smooth out any lateral temperature nonuniformity caused by the wire element. A black high impact polystyrene sheet $0.5 \mathrm{~mm}$ thick is bonded to the aluminium and has the opposite effect, in that it minimises lateral heat conduction along the surface thereby helping to retain the localised effect of temperature non-uniformity associated with the thermal footprints of a synthetic jet. The different layers of the composite are bonded together using a double-sided 3M adhesive tape to ensure good thermal contact and is inserted into the cavity so that the polystyrene sheet is flush to the lower test surface of the Perspex plate. To minimise 
heat losses through the back of the composite, a $5 \mathrm{~mm}$ air gap is sealed above the heater using a Perspex lid. A 5mm hole constitutes the SJA orifice through the composite, which has a total thickness equivalent to the orifice depth, $h$ of $5 \mathrm{~mm}$. The SJA cavity is mounted on the back of the composite on the surface of the heater.

A temperature-sensitive liquid crystal layer of approximately $50 \mu \mathrm{m}$ was applied on the polystyrene surface using an airbrush. A single coating of a binder (Hallcrest AQB-3) was applied on top of the liquid crystal layer and acts as a varnish to protect it from water. The liquid crystal used is BM/R22C5W supplied by Hallcrest and has an active colour bandwidth of $5^{\circ} \mathrm{C}$, showing red at $22^{\circ} \mathrm{C}$ and blue at $27^{\circ} \mathrm{C}$ (tolerances of quoted temperatures are $\pm 1^{\circ} \mathrm{C}$ ). In the experiment, the surface is heated to such an extent that the crystal shows blue colour under a laminar boundary layer. As the synthetic jet passes, its thermal footprint can be seen by a change in colour through the spectrum to red. The thermal footprints were captured with a standard colour video camera.

\subsection{Test Conditions}

In the present investigation, the non-dimensional flow characteristics of the synthetic jet were varied by changing the diaphragm operating conditions (oscillation frequency, $f$ and peak-to-peak displacement, $\Delta$ ) and freestream conditions (namely, the freestream velocity, $\left.U_{\infty}\right)$. A LabVIEW virtual instrument was used for signal generation of a sinusoidal waveform at a specified diaphragm oscillation frequency, which was sent to the permanent magnetic shaker via an ADC device. The diaphragm displacement was measured using an eddy current displacement sensor and read into the virtual instrument. The time-averaged blowing velocity over the entire cycle was evaluated from Eq. (2), which is applicable for incompressible flows. Equation (2) was validated 
from hotwire measurements by Li and Zhong (2005) with an uncertainty of 9.7\% between the theoretical and measured value of $\bar{U}_{o}$.

The freestream velocity was measured using a Nixon Streamflo 403 vane anemometer, which has an accuracy of $\pm 2 \%$ of the flow velocity. The undisturbed boundary layer velocity profiles were measured using PIV. To facilitate measurements, the freestream flow was seeded with hollow glass particles with a mean diameter of $10 \mu \mathrm{m}$ and a density of $1100 \mathrm{~kg} / \mathrm{m}^{3}$. The settling velocity of the particles is approximately $5 \mu \mathrm{m} / \mathrm{s}$ thus suggesting that the particles will follow the motion of the flow with high fidelity. The accuracy of the PIV measurements was assessed from continuity in accordance with the method outlined by Chang et al. (2001), which yielded a maximum uncertainty of $4.8 \%$. A light sheet approximately $1 \mathrm{~mm}$ in width was generated by a $5 \mathrm{~W}$ Argon ion laser to illuminate the flow field along a streamwise plane, which bisects the SJA orifice along its centreline. A Photron Ultima APX camera with a resolution of $1024 \times 1024$ pixels was used to capture images in a field of view of $25 \mathrm{~mm} \times 25 \mathrm{~mm}$. Corresponding vectors were resolved using a two-frame cross-correlation algorithm in which, a $32 \times 32$ pixel interrogation area with an overlap ratio of $50 \%$ was chosen giving a spatial resolution of $0.39 \mathrm{~mm}$. Long time-averaged velocity profiles were obtained over a recording time of 24 seconds. Comparison of the undisturbed boundary layer velocity profile with the Blasius solution at $U_{\infty}=0.05 \mathrm{~m} / \mathrm{s}$ and $U_{\infty}=0.1 \mathrm{~m} / \mathrm{s}$ along a streamwise centreline location of 5 orifice diameters upstream of the orifice is shown in Fig. 6. The good agreement confirms the zero-pressure gradient nature of the laminar boundary layer.

A summary of all the test cases is shown in Table 1. The dimensionless stroke length, $L$ varies between 0.8 and 5.1, Reynolds number, $\operatorname{Re}_{L}$ between 16 and 658, velocity ratio, $V R$ between 0.04 and 0.7 and Strouhal number, $S t$ between 0.05 and 0.2. 
For the two values of $U_{\infty}$ tested, $0.05 \mathrm{~m} / \mathrm{s}$ and $0.1 \mathrm{~m} / \mathrm{s}$, the respective changes in $d(3.6$ and 2.3) and $c_{f}\left(4.13 \times 10^{-3}\right.$ and $\left.2.65 \times 10^{-3}\right)$ obtained from the velocity profiles in Fig. 6 were relatively small such that their effects were considered secondary to changes in the other aforementioned parameters. Thus, the focus of the parameter space (Eq. (7)) with respect to the results in the next section is on $L, V R$ and $\mathrm{Re}_{L}$.

\section{RESULTS AND DISCUSSION}

\subsection{Near Wall Effect of Vortical Structures}

In the present study three main types of vortical structures have been identified from the interaction of a synthetic jet with a zero pressure gradient laminar boundary layer, namely hairpin vortex, stretched vortex ring and distorted vortex ring. These are similar to those observed by Zhong et al. (2005). In order to differentiate these structures by their impact on the flow in the near wall region where flow control is ultimately desired, liquid crystal images are correlated with the patterns observed by dye visualisation.

Stereoscopic dye visualisation and corresponding surface liquid crystal data of a synthetic jet produced at $V R=0.14, \operatorname{Re}_{L}=46$ and $L=1.4$ are shown in Fig. 7(a). It can be seen from the dye visualisation that under such low $V R$, elongated hairpin vortices are formed out of the orifice. The structures remain embedded within the near-wall region of the boundary layer on account of their low $V R$. As such, the level of shear in the boundary layer plays an important role in that the counter-rotating legs of the hairpins experience significant stretching as they propagate downstream. This is represented by the trace of the structure as seen with the dye, which appears as a thin line thus suggesting that the vortex tube is highly stretched. It may also be observed from both side and surface views that the counter-rotating legs of the hairpins can be traced back to the orifice from which they were formed, suggesting that the suction stroke is 
dominant for this type of structure (equating to a small stroke length). In addition to the hairpin structures, the surface view shows the development of two dye concentrations, which appear laterally to either side of the dark concentration created by the counterrotating legs of the hairpin vortices.

Similar observations were made by Acarlar and Smith (1987) in their study of hairpin vortices in a laminar boundary layer, where the dye concentrations were shown to be a secondary or longitudinal vortex pair not too dissimilar to the horseshoe-type vortex observed to form at end wall junctures. The formation of the longitudinal vortex pair is characterisitc of hairpin vortices formed at low injection rates, or as in this case, low $V R$ and are created by the inrush of fluid outboard of the counter-rotating hairpin legs.

Due to the weak nature of the structures caused by a low $\operatorname{Re}_{L}$, their ability to affect the near wall region appears limited, as confirmed by the corresponding liquid crystal image (however, one also needs to consider the development of the thermal boundary layer along the wall, which results in an increasing surface temperature downstream thereby making it more difficult to differentiate the thermal footprints with downstream distance).

Within $6 D_{o}$ of the orifice there appears three dark (red) streaks representing a drop in the local surface temperature due to a relatively high level of convective heat transfer associated with the passing structures. By Reynolds analogy, a local increase in heat transfer correlates to a local increase in fluid momentum. The two outer streaks are associated with the counter-rotating legs of the hairpins, which have 'common flow' upwards such that higher momentum fluid is brought towards the wall outboard of the legs. Conversely, the centre streak is associated with the longitudinal vortices, which have 'common flow' downwards (as shown by Acarlar and Smith, 1987) and from Fig. 
7 (a) are seen to form inboard of the hairpin vortex within $4 D_{o}$ of the orifice (note the two dye lines pertaining to the longitudinal vortices in between the hairpin legs in the near field region). Hence higher momentum fluid is brought towards the wall inboard of the longitudinal vortices resulting in a higher heat transfer region.

Figure 7(b) shows the formation and advection of hairpin vortices at $V R=0.19$, $\operatorname{Re}_{L}=89$ and $L=1.9$. Due to an increase in $\operatorname{Re}_{L}$, the strength of the hairpins appear greater than those in Fig. 7(a), as evidently shown by the vortex tube pertaining to the head and legs of the structure. It is also observed that the longitudinal vortices have completely diminished. Both types of hairpin vortices with and without the longitudinal vortices are collectively grouped as 'hairpin vortices' since the presence of a hairpin vortex is the dominant feature in both cases.

Figure 8(a) shows the corresponding development of the hairpin vortex as it is formed out of the orifice. The vortical structure produced by the synthetic jet is seen to experience an asymmetric roll up, similar to the findings of Zhong et al. (2005). Since the dimensionless stroke, $L$, for this case is quite small, the structure does not move sufficiently far from the orifice before the suction stroke begins. Consequently, the onset of suction inhibits roll up on the upstream side whilst the downstream branch is retained and strengthened by the vorticity in the boundary layer thereby resulting in an asymmetric structure. It is therefore evident that suction significantly influences the formation of these structures. The stereoscopic dye image reveals that the counterrotating legs of successive hairpins are connected with each other and are traceable back to the orifice, the effect of which can also be attributed to suction.

As the structures move downstream, as well as experiencing a high degree of stretching, the heads undergo a lifting movement from the wall due to self induction. It is found that the hairpin structures produce two streamwise streaks of high heat transfer 
that persist downstream. As in Fig. 7(a), the nature of these footprints can be attributed to the counter-rotating legs. As the legs of the hairpins rotate, low momentum fluid adjacent to the wall accumulates between them generating a region of low heat transfer (corresponding to the light (blue) region between the streamwise streaks). Outboard of the legs, higher momentum fluid is brought towards the wall, thereby generating two streamwise regions of high heat transfer that correspond to the dark (red) streaks produced by the liquid crystal.

Figure 7(c) shows the formation and advection of a stretched vortex ring at $V R$ $=0.27, \operatorname{Re}_{L}=182$ and $L=2.7$. Similarly, a close-up development of the structure out of the orifice is shown in Fig. 8(b). A rollup on the upstream side of the vortex is observed, which is significantly weakened by the vorticity in the boundary layer. Conversely, the strength of the downstream branch becomes intensified due to stretching. Anticlockwise tilting is also evident by the motion of the vortex head as the structure convects downstream. Based on the spinning solid cylinder concept suggested by Chang and Vakili (1995), Zhong et al. (2005) attributes this behaviour to the moment of the Magnus forces acting on the vortex rollers. The structure as shown by the dye pattern differs from the hairpin vortices in two ways. Firstly, the vortex rollers in the head of the structure are more prevalent (as shown in Figs. 7(c) and 8(b)), which may be attributed to an increase in $\mathrm{Re}_{L}$. Secondly, the counter-rotating legs of the structure detach away from the wall and also from the orifice, suggesting that for this stroke length of $L=2.7$, the effect of suction is minimal. Further observations show that the structures have a steeper trajectory than the hairpin vortices on account of their higher $V R$, which causes the structures to eventually penetrate out of the boundary layer.

Nevertheless, the near wall effect of these structures is qualitatively very similar to that which is observed for the hairpins, since the counter-rotating legs still sweep 
over the wall due to a degree of tilting. Two streamwise streaks of high heat transfer produced by the counter rotating legs are evident. Close observation reveals the width of these streamwise streaks is slightly greater than those produced by the hairpins. This could be attributed to the higher strength of the stretched vortex rings. Moreover, since the stretched vortex rings are stronger and further away from the wall, the counterrotating legs bring in higher momentum fluid from the surroundings than those of the hairpin vortices, thereby resulting in an increased rate of heat transfer along the wall. As in the work of Zhong et al. (2005), this type of structure is regarded as an intermediate case between those structures than remain embedded in the boundary layer, as previously described and those that rapidly penetrate it, as described below.

Figure 7(d) shows the advection of a distorted vortex ring out of the boundary layer (where $V R=0.51, \operatorname{Re}_{L}=658$ and $L=5.1$ ). The formation of the structure out of the orifice (Fig. 8(c)) shows that it initially experiences a symmetrical roll up, since the strength of the jet is increased relative to the freestream, with the ensuing vortical structure penetrating the boundary layer. Consequently, boundary layer shear has little influence on this structure unlike the previous cases, which experience a large degree of stretching due to their long resident time in the boundary layer.The structure, as shown in Fig. 8(c) becomes increasingly incoherent with time and the formation of secondary vortices can eventually be seen shedding from the primary vortex ring. The formation of these secondary vortices indicates that a limit in the circulation that can be contained within the primary vortex has been reached. For this case, the dimensionless stroke length is above the threshold of $L=4$ for the shedding of secondary vortices found for synthetic jets in quiescent conditions (Jabbal et al., 2006).

The corresponding thermal footprint produced by the distorted vortex ring is distinctly different to those observed for the hairpin vortices and stretched vortex rings. 
In this case, there is a single dark (red) streak of high heat transfer downstream of the orifice. In the case of Fig. 7(d), it is believed that the region of high heat transfer is produced by the unsteady wake of the jet, which protrudes the boundary layer. For a further increase in $V R$ it is expected that the near wall effect of the synthetic jet will diminish.

\subsection{Correlation of Vortical Structures to the Delayed Separation on a Cylinder}

Figure 9 shows a magnified view of the surface oil flow pattern generated by the interaction of a synthetic jet with the separation line on a circular cylinder, as previously seen in Fig. 2. The streaklines reveal a clear footprint associated with the development of a streamwise vortex pair downstream of the SJA orifice, which interacts with the separation. A flow topology of the streaklines, as presented by Crook (2002) is included for clarity. Based on the similarity in their thermal footprints to the flow pattern revealed by the oil flow on the $2 \mathrm{D}$ cylinder model, it is believed that either the hairpin vortices or the highly stretched vortex rings are responsible for producing the well defined streamwise vortex pairs that delayed flow separation on the $2 \mathrm{D}$ circular cylinder.

In addition to the streamwise tracks produced by the vortex pair, the flow topology downstream of the actuator orifice reveals the presence of a wedge region embedded between the streamwise vortices. Crook (2002) offered no explanation to the cause of this wedge region. However, a possible hypothesis for the wedge may be provided from Fig. 7(a) in which, a third streak embedded between the two streaks of high heat transfer is observed. As previously described the middle streak, which is attributed to the 'common flow' down induced by the longitudinal vortices that initially form inboard of the hairpin legs before moving laterally outboard of them, appears to 
give rise to a wedge-like pattern embedded between the vortices. This hypothesis seems to lend further support to the belief that the interaction of a synthetic jet with a laminar and turbulent boundary layer is essentially the same. As such, based on their near wall persistency and similar qualitative effects along the wall, both hairpin vortices (with and without a longitudinal vortex pair) and stretched vortex rings appear potentially useful.

\subsection{Parameter Mapping of Vortical Structures}

Having identified the possible types of vortical structures that were responsible in delaying flow separation on the cylinder, it is useful to establish the conditions that ensure the formation of such structures. To undertake this task, it was necessary to correlate all test cases to a single parameter map based on the key dimensionless parameters of synthetic jets in a boundary layer defined according to Eq. (7). As such, the test cases shown in Table 1 were plotted as a function of $L, V R$ and $\operatorname{Re}_{L}$ to give the surface contour plot in Fig. 10 in which, the gradient of the $L-V R$ plane is equivalent to the Strouhal number, $S t$ (Eq. (6a)).

From Fig. 10, it can be seen that there is a minimum threshold in the stroke length of $L \approx 1$ and $V R \approx 0.1$, which is required for vortex formation in a boundary layer. For the vortical structures believed to be responsible for the separation delay on the cylinder, it is possible to establish useful ranges in $L, V R$ and $\operatorname{Re}_{L}$ required for their formation. Fig. 10 confirms the importance of suction and a relatively small jet-tofreestream velocity ratio for the formation of hairpin vortices where the range in $L$ is limited to $1<L<3.5$ for velocity ratios of $0.1<V R<0.32$ and Reynolds numbers of $29<\operatorname{Re}_{L}<308$. For stretched vortex rings, where the influence of suction diminishes, the range in $L, V R$ and $\operatorname{Re}_{L}$ required for their formation is approximately $2.7<L<5.1$, $0.2<V R<0.38$ and $182<\operatorname{Re}_{L}<658$ respectively. The intermediate range of $L$ and $V R$ 
ensure roll up of the upstream vortex branch coupled with substantial resident time in the boundary layer. Finally for the distorted vortex rings, which are not considered to be responsible for the delayed separation on the cylinder due to their short resident time in the boundary layer and dissimilar footprints, $L>1.9, V R>0.38$ and $\operatorname{Re}_{L}>179$. The high threshold of $V R$ confirms that these structures penetrate the boundary layer over a short distance downstream.

From the contour plot it may be seen that limiting $V R$ for a particular type of structure results in an extension to the range of $L$ over which the structure is formed. This is true for both hairpin vortices and stretched vortex rings and suggests that the conditions for enhancing the flow control effectiveness of synthetic jets by the generation of vortical structures of relatively high strength that persist in the near wall region depends on limiting $V R$ (ideally $<0.38$ for the current set of cases) whilst increasing $L$ and hence $\operatorname{Re}_{L}$. This assessment also correlates with the previously defined scaling law derived by Raju et al. (2005), which shows that the vorticity flux of a synthetic jet embedded in a zero-pressure gradient laminar boundary layer varies as a linear function of $L$ and $V R^{-1}$. In the present study for a fixed SJA geometry and given set of freestream conditions, $L$ is a function of the peak-to-peak diaphragm displacement, $\Delta$ (Eq. (1)), $\operatorname{Re}_{L}$ a function of the diaphragm oscillation frequency, $f$ and $\Delta^{2}$ and $V R$ a function of $f$ and $\Delta$ (Eq. (5)). Thus, the outlined mechanism for SJA effectiveness may be achieved by increasing $\Delta$ whilst reducing $f$, thereby decreasing both $S t$ and $S$. However, there is a compromise to be attained between reducing the actuation frequency for maximising vortex strength near the wall and ensuring a Stokes number greater than 10 for vortex roll up (Guo and Zhong, 2005).

The parameter space for the synthetic jet array implemented on the cylinder model suggests that the delayed separation shown in Fig. 9 was achieved via the 
aforementioned route for flow control, in which the Strouhal number was estimated to be 0.036 (where the local external velocity of the cylinder was assumed to be twice that of the freestream), a $V R$ of 0.25 and $L$ approximately 7 . Although this dataset is beyond the range of the contour plot shown in Fig. 10, it is anticipated that it would yield the formation of a highly stretched, streamwise-aligned hairpin vortex or stretched vortex ring. For a fully turbulent boundary layer however, the types of synthetic jet structures formed are expected to remain as those identified but the magnitude of $V R, L$ and $\operatorname{Re}_{L}$ at which these structures are produced is likely to shift.

\section{CONCLUSIONS}

An experimental investigation to obtain the qualitative near wall effect of synthetic jets in a laminar boundary layer has been undertaken for the purpose of identifying the types of vortical structures likely to have delayed separation on a 2D circular cylinder model described herein. Dye visualisation of synthetic jets in conjunction with liquid crystal visualisation along the wall was conducted for a range of actuator operating and freestream conditions. From this investigation, it has been found that

- Three types of vortical structures have been identified from the interaction of a synthetic jet with a zero pressure gradient laminar boundary layer - hairpin vortices (with and without secondary longitudinal vortices), stretched vortex rings and distorted vortex rings. These structures differ from each other primarily in terms of vorticity strength and in the time they are resident in the near wall region.

- The thermal footprints of the hairpin vortices and stretched vortex rings revealed a marked similarity with the oil flow pattern generated on a 2D circular cylinder 
model in terms of the wedge region and streamwise tracks, hence suggesting that either of these structures was responsible for the delayed separation on the cylinder.

- Conditions were established for the formation of these structures in non-dimensional parameter space - hairpin vortices: $1<L<3.5,0.1<V R<0.32$ and $29<\operatorname{Re}_{L}<308$; stretched vortex rings: $2.7<L<5.1,0.2<V R<0.38$ and $182<\operatorname{Re}_{L}<658$.

Work is currently being undertaken to obtain quantitative measurements for an in-depth analysis of the near wall effects of synthetic jets in a boundary layer. High frame rate PIV will be utilised in streamwise planes to provide real-time velocity profile and wall shear stress measurements. It is anticipated that the findings from these investigations will allow for a potential identification of the vortical structures effective for flow separation control.

\section{ACKNOWLEDGEMENTS}

The first author, Mark Jabbal, would like to acknowledge the financial support from the EPSRC for his $\mathrm{PhD}$ study. The authors would also like to thank the technician staff at the Goldstein Laboratory for their support. Helpful discussions with Mr Costas Marrakos regarding the PIV uncertainty analysis are acknowledged.

\section{REFERENCES}

Acarlar, M.S., Smith, C.R., 1987. A study of hairpin vortices in a laminar boundary layer. Part 2. Hairpin vortices generated by fluid injection. Journal of Fluid Mechanics 175, 43-83.

Cater, J.E., Soria, J., 2002. The evolution of round zero-net-mass-flux jets. Journal of Fluid Mechanics 472, 167-200. 
Chang, K.-A., Hsu, T.-J., Liu, P.L.F., 2001.Vortex generation and evolution in water waves propagating over a submerged rectangular obsatacle. Part 1. Solitary waves. Coastal Engineering 44, 13-36.

Chang, Y.K., Vakili, A.D., 1995. Dynamics of vortex rings in crossflow. Physics of Fluids 7 (7), 15831597.

Crook, A. 2002. The control of turbulent flows using synthetic jets. PhD Thesis, University of Manchester, UK.

Crook, A., Wood, N.J., 2001. Measurements and visualisations of synthetic jets. In: Proceedings of $39^{\text {th }}$ AIAA Aerospace Sciences Meeting and Exhibit, Reno, NV, USA, paper 2001-0145.

Gharib, M., Rambod, E., Shariff, K., 1998. A universal time scale for vortex ring formation. Journal of Fluid Mechanics 360, 121-140.

Gilarranz, J.L., Rediniotis, O.K., 2001. Compact, high-power synthetic jet actuators for flow separation control. In: Proceedings of $39^{\text {th }}$ AIAA Aerospace Sciences Meeting and Exhibit, Reno, NV, USA, paper 2001-0737.

Glezer, A. 1988. The formation of vortex rings. Physics of Fluids 31 (9), 3532-3542.

Glezer, A., Amitay, M., 2002. Synthetic jets. Annual Review of Fluid Mechanics 34, 503-529.

Guo, F., Zhong, S., 2006. A PIV investigation of of the characteristics of micro-scale synthetic jets. In: Proceedings of $3^{\text {rd }}$ AIAA Flow Control Conference, San Francisco, CA, USA, paper 2006-3183.

Holman, R., Utturkar, Y., Mittal, R., Smith B.L., Cattafesta, L., 2005. Formation criterion for synthetic jets. AIAA Journal 43 (10), 2110-2116.

Jabbal, M., Wu, J., Zhong, S., 2006. The performance of round synthetic jets in quiescent flow. The Aeronautical Journal 110 (1108), 385-393.

James, R.D., Jacobs, J.W., Glezer, A., 1996. A round turbulent jet produced by an oscillating diaphragm. Physics of Fluids 8 (9), 2484-2495.

Li, M., Zhong, S., 2005. Heat transfer and flow characteristics of impinging synthetic jets. In: Proceedings of $9^{\text {th }}$ UK National Heat Transfer Conference, Manchester, UK, paper UKHTC9-57. Milanovic, I.M., Zaman, K.B.M.Q., 2005. Synthetic jets in crossflow. AIAA Journal 43 (5), 929-940. Narasimha, S., Prasad, S.N., 1994. Leading edge shape for flat plate boundary layer studies. Experiments in Fluids 17 (5), 358-360. 
Raju, R., Mittal, R., Gallas, Q., Cattafesta, L., 2005. Scaling of vorticity flux and entrance length effects in zero-net mass-flux devices. In: Proceedings of $35^{\text {th }}$ AIAA Fluid Dynamics Conference and Exhibit, Toronto, ON, Canada, paper 2005-4751.

Smith, B.L., Glezer, A., 1998. The formation and evolution of synthetic jets. Physics of Fluids 10 (9), 2281-2297.

Tang, H., Zhong, S., 2006. Incompressible flow model of synthetic jet actuators. AIAA Journal 44 (4), 908-912.

Zhang, S., Bremhorst, K., Turner, J.T., 2003. Stereoscopic visualisation of the large-scale structures in an aerodynamically excited turbulent jet. In: Proceedings of $7^{\text {th }}$ Triennial Symposium on Fluid Control, Measurement and Visualisation, Sorrento, Italy.

Zhong, S., Kittichaikan, C., Hodson, H.P., Ireland, P.T., 2000. Visualisation of turbulent spots under the influence of adverse pressure gradients. Experiments in Fluids 28, 385-393.

Zhong, S., Millet, F., Wood, N.J., 2005. The behaviour of circular synthetic jets in a laminar boundary layer. The Aeronautical Journal 109 (1100), 461-470. 
Table 1. Experimental parameters

\begin{tabular}{|l|lll|lll|lll|lll|}
\hline \multicolumn{7}{|c|}{$U_{\infty}=0.05 \mathrm{~m} / \mathrm{s}$} & \multicolumn{5}{c|}{$U_{\infty}=0.1 \mathrm{~m} / \mathrm{s}$} \\
\hline$L$ & $\operatorname{Re}_{L}$ & $V R$ & $S t$ & $\operatorname{Re}_{L}$ & $V R$ & $S t$ & $\operatorname{Re}_{L}$ & $V R$ & $S t$ & $\operatorname{Re}_{L}$ & $V R$ & $S t$ \\
\hline 0.8 & 16 & 0.08 & & 33 & 0.16 & & 16 & 0.04 & & 33 & 0.08 & \\
1.1 & 29 & 0.11 & & 58 & 0.22 & & 29 & 0.05 & & 58 & 0.11 & \\
1.4 & 46 & 0.14 & & 91 & 0.27 & & 46 & 0.07 & & 91 & 0.14 & \\
1.9 & 89 & 0.19 & & 179 & 0.38 & & 89 & 0.09 & & 179 & 0.19 & \\
2.2 & 117 & 0.22 & 0.1 & 233 & 0.43 & 0.2 & 117 & 0.11 & 0.05 & 233 & 0.22 & 0.1 \\
2.7 & 182 & 0.27 & & 365 & 0.54 & & 182 & 0.14 & & 365 & 0.27 & \\
3.5 & 308 & 0.35 & & 616 & 0.70 & & 308 & 0.18 & & 616 & 0.35 & \\
4.1 & 410 & 0.41 & & & & & 410 & 0.20 & & & & \\
5.1 & 658 & 0.51 & & & & & 658 & 0.26 & & & & \\
\hline
\end{tabular}




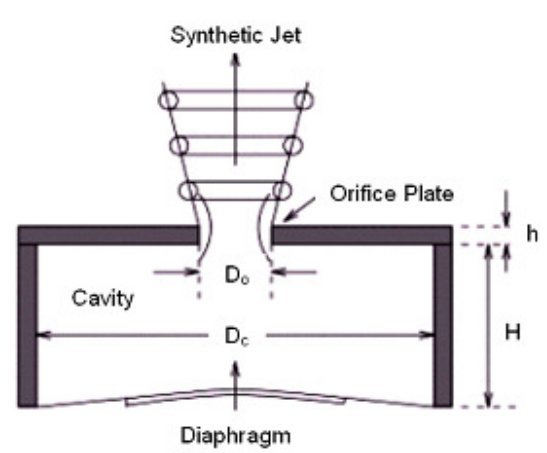

Figure 1. Schematic of a SJA

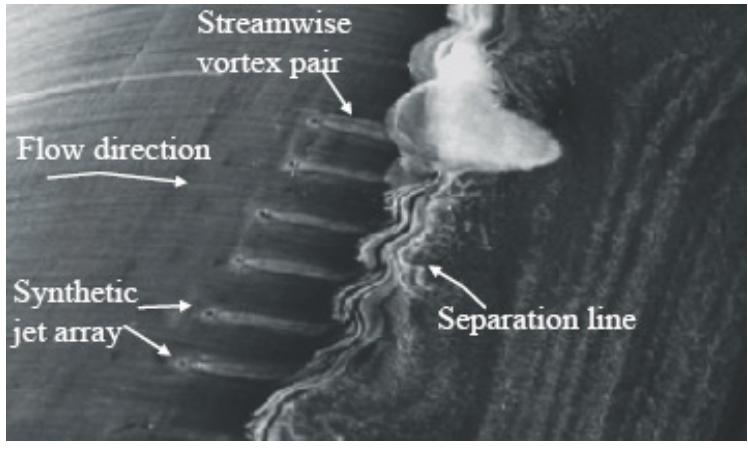

Figure 2. Demonstration of flow separation control (Crook and Wood, 2001)

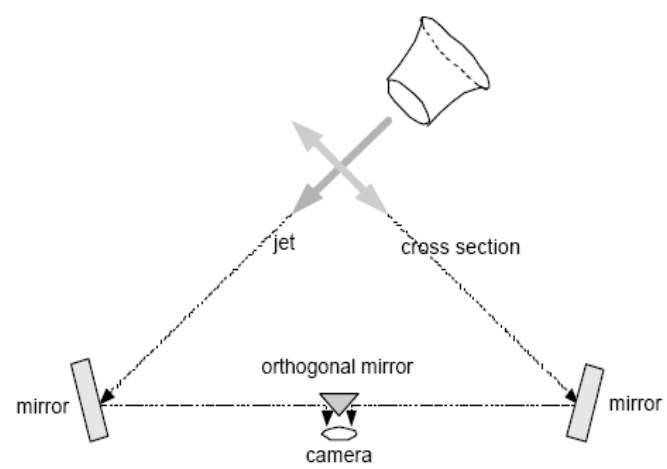

Figure 4. Stereoscopic viewing system for dye visualization (Zhang et al., 2003)

(b) (a)

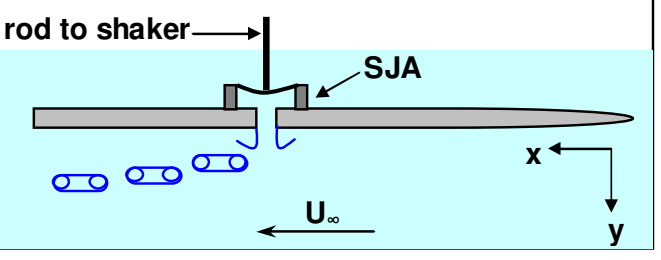

Figure 3. Schematic sketch of test section
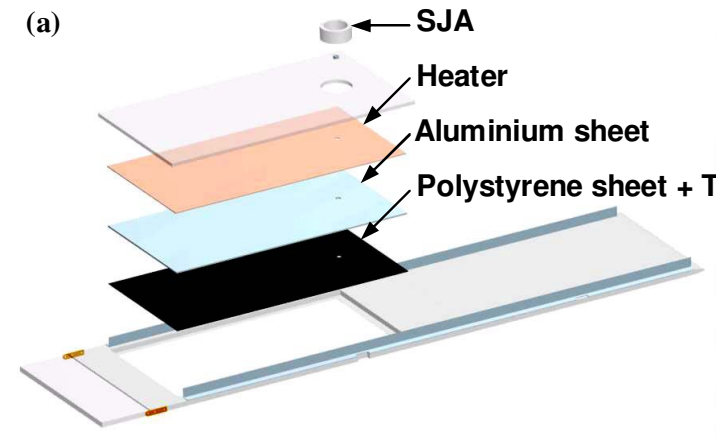

(b)

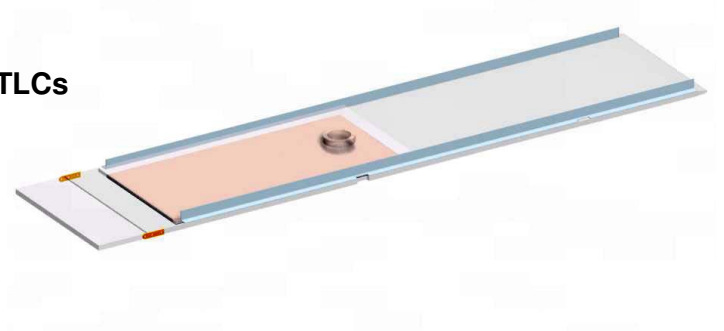

Figure 5. (a) Modular construction of flat plate embedded with heat transfer sensing system and (b) plate in its complete form 

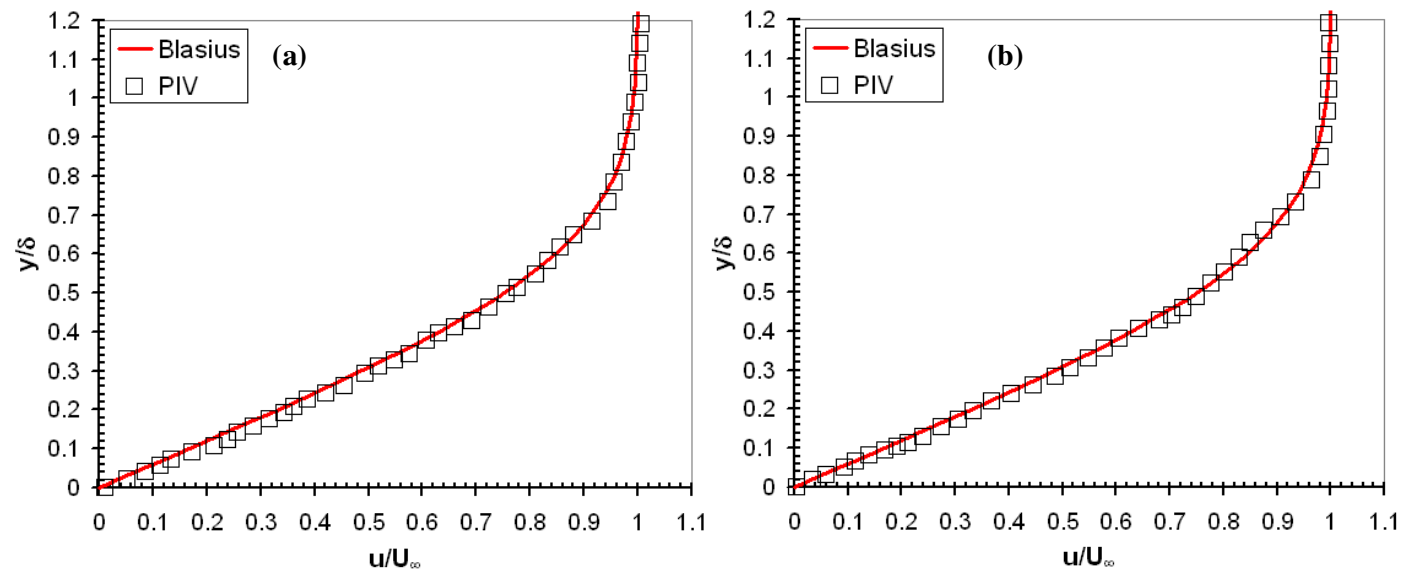

Figure 6. Undisturbed boundary layer velocity profiles along a streamwise centreline location at $\mathrm{x} / D_{o}=-5$ for (a) $U_{\infty}=0.05 \mathrm{~m} / \mathrm{s}$ and (b) $U_{\infty}=0.1 \mathrm{~m} / \mathrm{s}$ 


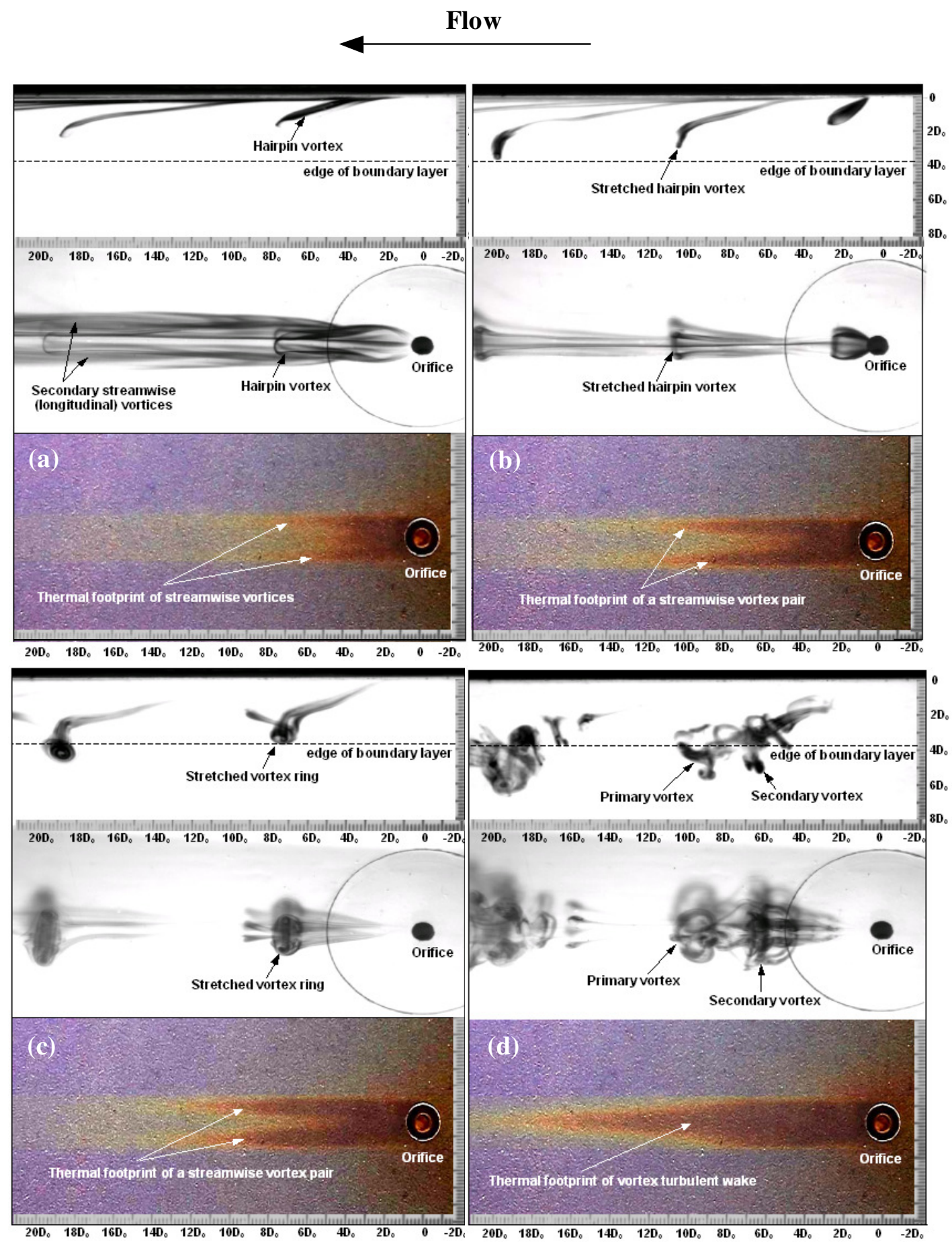

Figure 7. Stereoscopic dye and surface liquid crystal images of a synthetic jet at (a) $V R=0.14$, $\operatorname{Re}_{L}=46 \& L=1.4$, (b) $V R=0.19, \operatorname{Re}_{L}=89 \& L=1.9$, (c) $V R=0.27, \operatorname{Re}_{L}=182 \& L=2.7$ and (d) $V R$ $=0.51, \operatorname{Re}_{L}=658 \& L=5.1-S t=0.1$ and $d \approx 4$ for all cases 


\section{Flow}

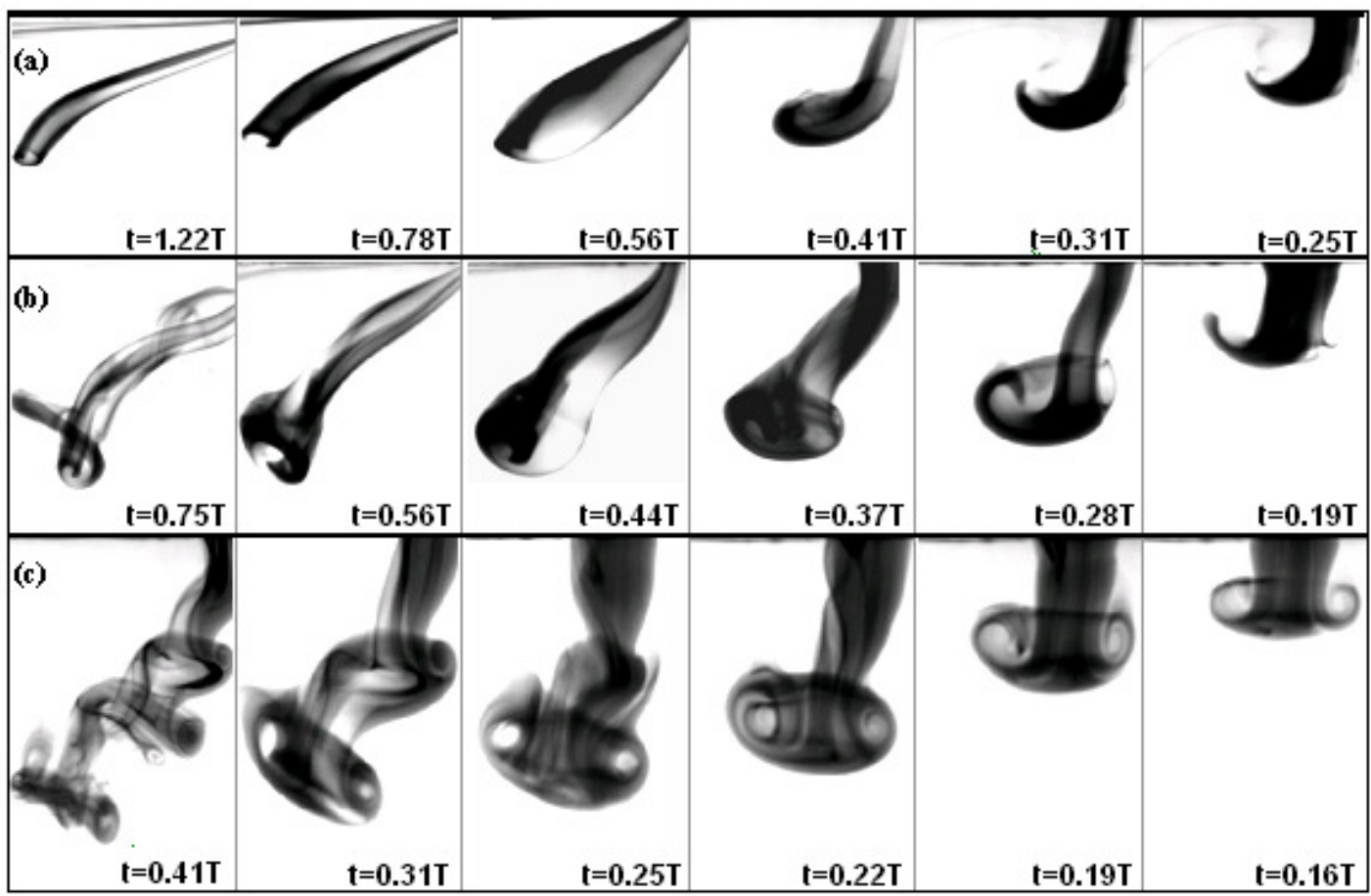

Figure 8. Close-up view of synthetic jet formation and roll up for (a) hairpin vortices, (b) stretched vortex rings and (c) distorted vortex rings

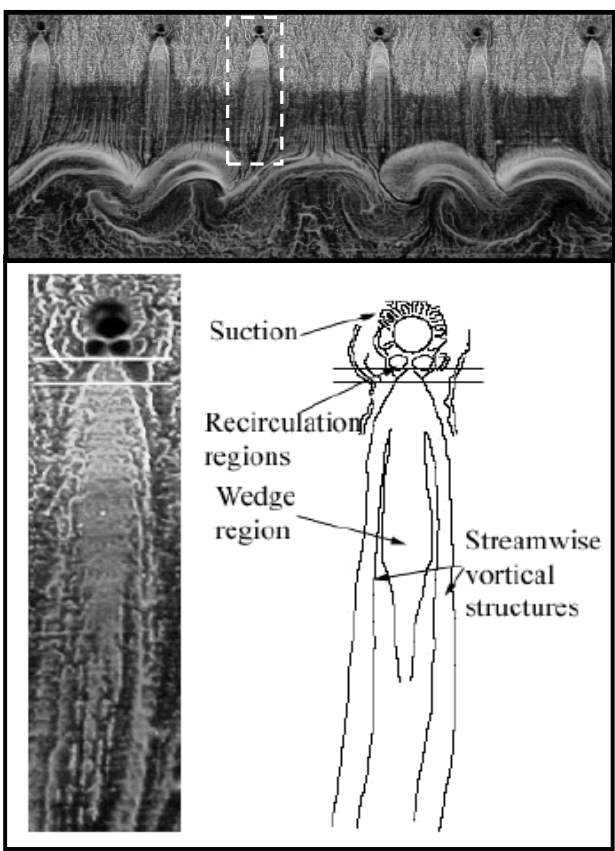

Figure 9. Oil streaklines and corresponding flow topology of a streamwise vortex pair produced by a synthetic jet interacting with flow separation (Crook 2002) 

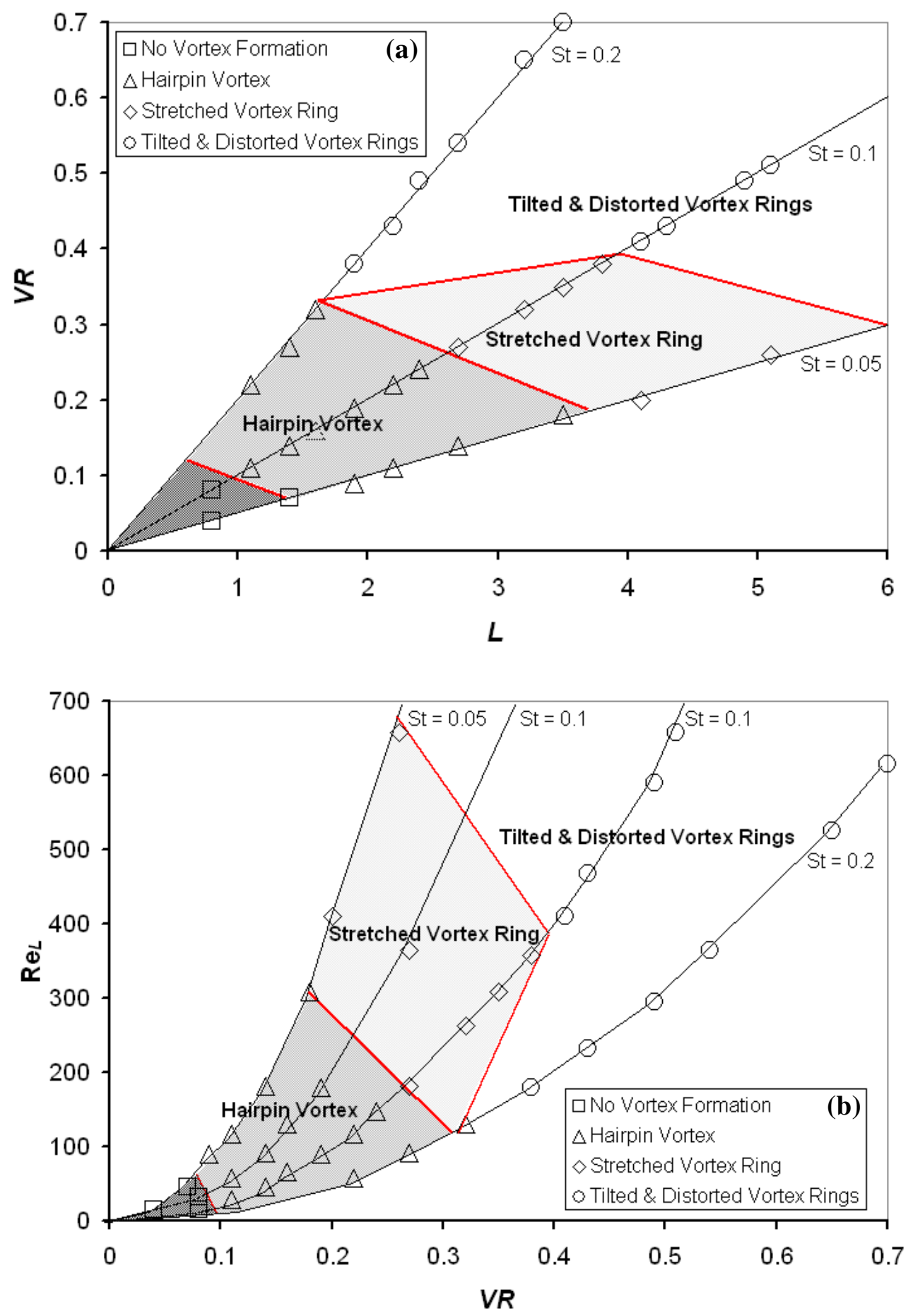

Figure 10. (a) $V R-L$ and (b) $\operatorname{Re}_{L^{-}}-V R$ parameter space of the different vortical structures seen as a result of the interaction between a synthetic jet and a boundary layer 